\title{
Aum Shinrikyô : secte et violence (Partie 2)
}

\section{Sylvaine Trinh}

\section{OpenEdition}

\section{Journals}

\section{Édition électronique}

URL : http://journals.openedition.org/conflits/720

DOI : $10.4000 /$ conflits. 720

ISSN : $1777-5345$

Éditeur :

CCLS - Centre d'études sur les conflits lilberté et sécurité, L'Harmattan

\section{Édition imprimée}

Date de publication : 15 octobre 1998

ISSN : 1157-996X

\section{Référence électronique}

Sylvaine Trinh, « Aum Shinrikyô : secte et violence (Partie 2) », Cultures \& Conflits [En ligne], 29-30 | automne-hiver 1998, mis en ligne le 16 mars 2006, consulté le 30 mars 2021. URL : http:// journals.openedition.org/conflits/720 ; DOI : https://doi.org/10.4000/conflits.720

Ce document a été généré automatiquement le 30 mars 2021.

Creative Commons License 


\title{
Aum Shinrikyô : secte et violence (Partie 2)
}

\author{
Sylvaine Trinh
}

1 La tentation de l'action politico-religieuse

2 En août 1989, lors d'une discussion à Fujinomiya, Asahara Shôkô prend la décision d'engager l'organisation dans la course aux législatives. En janvier 1990, il annonce publiquement que lui-même et vingt-cinq de ses condisciples seront les candidats du Shinritô [Parti de la Vérité, fondé par la secte] aux élections qui doivent avoir lieu le mois suivant.

3 Qu'une secte se lance dans l'action politique n'est pas un fait nouveau au Japon. Seichô no Ie s'est, dès la fin de la guerre, activement engagée dans l'action politique et est devenue à partir de 1958 un soutien reconnu de l'aile droite du Parti-LibéralDémocrate. Les liens puissants et politiquement déterminants qu'entretient la Soka Gakkai avec des partis comme le Komeitô, puis le Shinshintô sont déjà anciens, bien connus et relativement clairs.

4 Dès l'annonce des candidatures, l'état-major de Aum Shinrikyô se comporte alors comme un parti politique. Il lui faut concevoir sa campagne et la financer. La secte collecte alors une somme modeste pour une campagne japonaise, mais importante pour un tout jeune parti : un million de yens est recueilli auprès de ses adeptes pour soutenir la campagne. Les candidats vont alors à la rencontre des électeurs, accompagnés d'un groupe plus ou moins important de disciples. Souvent tous vêtus de leurs vêtements religieux blancs, ils défilent dans les rues, organisent des meetings. Sans programme politique bien clair, ils annoncent un monde meilleur. Malgré leurs efforts, le nombre de voix portées sur chaque candidat est totalement dérisoire : aucun ne sort vainqueur. Ce revers cuisant provoque-t-il l'engrenage de la violence? N'est-ce pas en juin de cette même année que l'avocat Sakamoto commence à rassembler des preuves contre la secte ? L'échec a pu mettre en cause l'autorité du gourou au sein du mouvement, a pu faire naître des conflits internes et $a$, sans aucun doute, affaibli la situation financière de l'organisation. De nombreux observateurs désigneront ce moment comme déterminant pour expliquer le changement d'attitude de la secte. Lors des procès, 
plusieurs anciens disciples confirmeront cette interprétation. La tentative de retour au social par l'action politique n'a pas marché. Mais Aum Shinrikyô a-t-elle vraiment perdu?

5 Les efforts de communication portent en effet rapidement leurs fruits et l'entrée en campagne électorale donne une visibilité très nouvelle à la secte. Celle-ci passe par le soin qu'apporte Asahara Shôkô à son image publique. Il commence alors à être connu car il apparaît souvent sur les écrans de télévision, notamment au cours de débats religieux. Il a un certain talent que lui reconnaissent de nombreux auditeurs : tout en parlant toujours d'un ton posé, il sait placer ses attaques avec pertinence et déstabiliser ses adversaires sans abaisser le niveau de la discussion. Les thèmes religieux avaient jusqu'alors été peu abordés et ces débats suscitent l'intérêt du public et donc des producteurs d'émission. Son langage paraît accessible et moderne. Ses exemples sont démonstratifs. Son savoir-faire oratoire, la portée de ses critiques ou même le caractère original de ses pratiques autant que la forme des émissions poussent les auditeurs dans son camp. Le travail sur cette image positive construite à partir de connaissances religieuses s'intensifie à partir de 1989. Asahara Shôkô rédige, en tout cas signe, de nombreux textes que la secte publie. Ces textes visent la diffusion de son enseignement religieux. Certains décrivent l'enchaînement des pratiques. D'autres mettent à plat le dogme sur lequel repose l'existence de la secte. Plusieurs de ces textes existent dans une version anglaise.

6 La notoriété de Asahara Shôkô grandit. Alors que Aum Shinrikyô n'a été reconnue qu'en août 1989, le gourou voit de nombreuses portes s'ouvrir devant lui. Il est très sollicité pour dispenser des conférences. En particulier, il fait la tournée des universités les plus prestigieuses. Sans doute savoure-t-il le moment où il est invité par la plus prisée d'entre toutes, l'Université de Tokyo, qui l'avait refusé comme étudiant. Un exshukke raconte: " Ce 1er janvier 1991, commençaient les cours de Shinshû et j'allais successivement dans tous les meetings de Aum Shinrikyô, à l'Université du Tohoku, à l'université de Tokyo, à l'université de Kyoto, partout. Et nous [les étudiants] prenions tous ces invitations pour une grande affaire. A ce moment-là, Asahara présentait des perspectives tout à faits positives "1.

7 Quand survient l'attentat du métro, bien rares sont les Japonais qui n'ont jamais entendu parler de Asahara Shôkô.

L'affirmation du dogme et des pratiques

En 1989-1990, Asahara Shôkô donne un tour nouveau aux croyances de la secte. A partir d'avril 1990, il commence à prêcher auprès de ses disciples la nécessité d'une mort massive et sans discrimination, présentée comme seule solution pour sauver l'humanité puisque, ainsi, l'âme des morts peut se réincarner à un niveau supérieur. La référence incessante à des tantra précisément sélectionnés par le gourou, véritables formules incantatoires récitées en choeur à longueur de temps, est supposée légitimer l'échafaudage spirituel. Et la doctrine se développe de telle manière qu'elle appelle le châtiment de ceux qui s'opposeraient à l'organisation religieuse.

Nostradamus apparaît alors comme un personnage central dans les discours et dans les textes du gourou qui annonce la fin prochaine du monde. Comme lui, Asahara Shôkô se livre alors à des prédictions, qu'il rend publiques. En janvier 1990, dans un de ses prêches, il affirme que Aum Shinrikyô est prête à assumer sa place en tant que religion la plus élevée du monde et il prédit qu'elle sera attaquée par la police et par la société. Deviennent tout à fait essentielles les références à l'Apocalypse, à Nostradamus, aux 
prédictions et à Armagedon, qu'il considère comme un événement-lieu, et dont il annonce l'arrivée aux alentours de l'an 2000. Il n'y aura de salut que pour ceux qui auront suivi un entraînement physique et psychologique adapté pour résister aux armes qui seront utilisées pour Armagedon lors de la Troisième Guerre mondiale annoncée, que pour ceux qui auront suivi un enseignement spirituel très poussé : il n'y aura de salut que pour certains shukke de Aum Shinrikyô. La voie est difficile, ce que seul Asahara Shôkô a compris : " Sincèrement, il y a trop d'organisations religieuses qui disent : " il vous faut seulement la foi pour trouver le bonheur après la mort. Eh bien, en fait, ce n'est pas si simple "2. Shimazono Susumu s'appuie sur un texte de la secte, intitulé Kagirinaku tômeina sekai e no izanai [Invitation à un monde infiniment transparent], pour établir que le plan de sauvetage de l'humanité pensé par Aum Shinrikyô se précise dès $1988^{3}$.

11 A la même époque, à partir de 1989-1990, les rites deviennent plus sévères, les pratiques plus dures et plus intensives. En contrepartie, le nombre de shukke déclarés Illuminés par le gourou augmente. " Jésus-Christ était incapable de répandre la Loi, même sur la toute petite Judée. Si le Sauveur devait apparaître au moment de l'extinction de l'humanité, pourrait-il sauver seul tous les êtres? Bien sûr que non. Pour réussir, les disciples du Sauveur doivent avoir atteint un haut niveau spirituel. En d'autres mots, il doit y avoir beaucoup de saint-esprits. L'enseignement du Sauveur doit faire qu'il y ait beaucoup d'Illuminés. Exactement comme le fait Aum Shinrikyô. Et leur niveau spirituel doit être clairement défini "4.

12 Ainsi, si, au début, il s'agissait de développer des pouvoirs surhumains par la pratique du yoga, le but spirituel se complexifie par la suite. Par un approfondissement personnel à Asahara Shôkô, la doctrine de l'Illumination qui repose sur le bouddhisme exigerait un ascétisme extrême. Surtout, pour atteindre l'Illumination, il faut non seulement se détacher du monde réel, mais le rejeter. Et le seul moyen d'y parvenir est la méditation. Asahara Shôkô propose de raccourcir le chemin qui mène au salut: il suffit d'observer les rites et de suivre les initiations proposées par Aum Shinrikyô. Le fameux casque apparaît en 1990, appareillage conçu au sein de l'organisation pour lequel les premiers essais eurent lieu en 1989. Le port de cet appareil, muni d'électrodes censées relier les ondes émises par le cerveau du gourou au porteur du casque, devait permettre d'atteindre le même état de méditation que celui du maitre. Diverses techniques fondées sur la psychologie sont alors utilisées de manière intense, si bien que de nombreuses associations, les médias et beaucoup de personnalités dénonceront par la suite les pratiques de lavage et de contrôle des cerveaux de la secte. L'hôpital interne se structure autour de plusieurs médecins devenus shukke sous la direction de Hayashi Ikuo, personnage central au sein de Aum Shinrikyô.

13 Même si les pratiques internes à la secte ne sont pas connues à l'extérieur, son discours est tout à fait public. Bien que les candidats du Parti de la Vérité n'aient pas été élus, le lien social demeure autour du débat religieux entre divers mouvements, différentes religions et entre des éléments de la société et la secte. En même temps, il peut paraître étrange que le discours de la secte ait pu attirer aussi facilement le public. Hormis les éléments qui tiennent à la personnalité du gourou, il y a sans doute d'autres caractéristiques de la séduction qu'opérait Aum Shinrikyô. Ainsi, malgré la rationalisation qui a fortement imprégné ce pays, la population reste solidement attachée à des croyances de nature religieuse. D'autre part, il y a des phénomènes de mode très particuliers au Japon sur lesquels la secte a très bien su " surfer ". Par 
exemple, l'utilisation de l'adoration d'un chanteur disparu a déjà été évoquée. De même, la sur-utilisation des mots d'origine étrangère correspond à un goût très prononcé depuis quelques années pour les consonances qui évoquent l'exotisme: tantra, aum, Nostradamus, Armagedon... Les textes et les discours de la secte sont ainsi parsemés de mots chargés de mystère. Si le gourou police l'image de la secte vis-à-vis de l'extérieur, il bâtit aussi des fondations solides. que le mouvement religieux considérera comme un tournant dans sa courte histoire. Aum Shinrikyô organise à Ishigakijima, dans l'archipel d'Okinawa, un séminaire destiné à ses disciples. Et, cette fois, le succès est retentissant: environ cinq cents fidèles décident pendant le séminaire de rejoindre Aum Shinrikyô. Parmi eux, presque deux cents renoncent au monde et entrent dans la communauté des shukke. Au sein de la secte, on prendra souvent cette date comme référence : avant ou après Ishigakijima. Après avoir essayé de nouvelles formes de communication avec la campagne électorale, après avoir tenté en vain d'acquérir de nouveaux pouvoirs par des méthodes politiques et légales, et raté cette tentative de retour au social, le succès d'Ishigakijima sonne comme la Vraie reconnaissance non seulement des croyances de la secte, mais du gourou pour lequel ce rassemblement représente un succès personnel indéniable. 1989 et 1990 sont des années d'expansion : le nombre des shukke autant que celui des zaike augmente de manière importante.

17 Aum Shinrikyô s'installe. Le mouvement se rend propriétaire, à partir de 1989, de sept lots de terrain situés sur la commune de Kamikuishiki, dans la préfecture de Yamanashi, à une centaine de kilomètres de la capitale. Au pied du Mont Fuji, dans un lieu que les Japonais tiennent pour sacré, la région, parsemée de lacs, est très belle. La surface totale acquise est de $48000 \mathrm{~m}^{2}$. Combinant leurs compétences, les membres de la secte construisent eux-mêmes un bâtiment après l'autre. L'ensemble forme rapidement un véritable complexe. Les bâtiments les plus importants du point de vue de Aum Shinrikyô portent un nom: Satyam ${ }^{5}$, suivi d'un numéro. A cela s'ajoutent de nombreux entrepôts. Tous les bâtiments sont blancs. La secte fait de Kamikuishiki son principal lieu de culte: y seraient déposés des fragments sacrés d'os de Bouddha, rapportés du Sri-Lanka par Asahara Shôkô. Une image géante de Siva, déesse hindoue de la destruction et de la création très vénérée par le culte, contribue à sacraliser le site. D'autres bâtiments sont dans le district voisin de Fujigamine. Entre 1989 et 1994, trente bâtiments importants sont construits. De nombreux shukke résident sur le site. Certains de ces immeubles deviendront très célèbres : Satyam 6 , résidence de Asahara Shôkô; Satyam 7, l'usine chimique où se fabriquait entre autres le sarin; Satyam 2, bureaux du gourou. L'ensemble de la propriété sera évalué à cent millions de yens. La communauté religieuse installée à Kamikuishiki vit en quasi-autarcie. Les shukke produisent eux-mêmes l'essentiel de leur alimentation et les enfants sont éduqués sur place. Onze disciples, dont six sont professeurs en titre, prennent en charge l'enseignement et utilisent les manuels nationaux. Les enfants suivent neuf heures de cours par jour, dont six consacrées aux disciplines classiques et trois à l'enseignement 
religieux. Ils regardent aussi régulièrement des programmes d'enseignement télévisés. Eduqués sous la responsabilité de disciples, ces enfants voient leurs parents biologiques plusieurs fois par semaine. Après l'attentat de Tokyo, cinquante-trois enfants âgés de deux à quatorze ans, seront trouvés à Kamikuishiki. La police en placera quarantequatre dans des institutions spécialisées de protection juvénile, les autres, c'est-à-dire une toute petite minorité, ayant été recueillis par des membres de leur famille ${ }^{6}$.

Kamikuishiki n'est pas le seul investissement immobilier de la secte. Pendant la même période, à travers l'ensemble du pays, Aum Shinrikyô se rend propriétaire, à partir de 1989, d'environ $300000 \mathrm{~m}^{2}$ de terrains, évalués à 2,25 milliards de yens. Ces terres sont réparties sur une trentaine de sites sur lesquels la secte établit peu à peu ses représentations locales; l'ensemble des bâtiments sera évalué à 1,33 milliard de yens. De plus, le Conseil municipal de Namino, préfecture de Kumamoto, doit encore verser à la secte qui lui a rendu des terrains 920 millions de yens. Le site sur lequel se trouve la branche d'Osaka est estimé à 360 millions de yens et le centre de formation de Tokyo à 150 millions. Même si l'éclatement de la bulle financière a rabaissé le prix du foncier à des niveaux plus raisonnables aujourd'hui qu'hier, l'importance de ces acquisitions montre l'aisance financière qu'avait acquise Aum Shinrikyô en peu de temps.

Pour Aum Shinrikyô, l'argent est une ressource capitale. Asahara Shôkô a de grands projets. Il est entouré de fidèles munis de diplômes de très haut niveau qui lui apportent des conseils dans tous les domaines. Le mouvement doit collecter des fonds, les gérer afin de réaliser au plus vite ses objectifs. Elle a des intérêts au sein de différentes entreprises dans des secteurs variés. Certains adeptes font don de leur société à la secte. D'autres hommes d'affaires devenus moines déclarent leur entreprise en faillite et transmettent la propriété à Aum Shinrikyô. L'ensemble constitue assez rapidement un petit réseau de petites et moyennes entreprises, dont les propriétaires, présidents et directeurs généraux sont des dirigeants de Aum Shinrikyô. L'entreprise Maha-Posya est ainsi spécialisée dans l'assemblage et la vente de matériels informatiques à travers une dizaine de filiales réparties sur le territoire national ${ }^{7}$. Il y a différentes sociétés de trading dont l'une, " La Belle Epoque ", installée à Osaka, approvisionnera la secte en matériel militaire. Hayakawa Kiyohide est président de World Unification Trade and Industry, société par laquelle transiteront aussi divers produits. Plusieurs restaurants, qui emploient des disciples, appartiennent à Aum Shinrikyô. Ces entreprises offrent à la secte une façade respectable. Mais il est probable que son réseau d'entreprises lui permet plus de fournir des emplois aux adeptes qui en ont besoin et de se procurer des matières premières pour servir ses projets que de collecter des fonds.

La richesse de la secte s'est surtout bâtie sur les dons des shukke. Takahashi Hidetoshi relate son arrivée en tant que shukke à Kamikuishiki : " Puis on me fit rédiger mes volontés. Comme j'étais devenu shukke, j'offrais la totalité de mes biens, mobiliers et immobiliers. Je précisais que c'était de mon plein gré et que je ne causerai pas le moindre problème à l'organisation religieuse. J'écrivais aussi que, en cas de décès, je souhaitais que le groupe prenne en charge mes funérailles. Quand j'eus terminé, j'apposais mon sceau "8.

21 Beaucoup a été écrit sur les tarifs pratiqués au sein de la secte où l'on ne parlait ni de prix, ni d'argent, mais d'offrande. Egawa Shôko évoque largement le fait que tout se vendait et s'achetait: " Des prospectus éparpillés sont vendus : 2 yens, la feuille de format B4, 1,5 yen la feuille en B5, [...] la vidéo d'un prêche de Asahara Shôkô coûte 15 
000 yens, la cassette vidéo 'Les secrets du yoga de l'Himalaya', 100000 yens, l'enregistrement de musique religieuse, de 10000 à 30000 yens, l'eau sacrée, 4000 yens les trois litres... En plus, il y a l'orientation personnalisée vers le yoga sacré pour 10000 yens de l'heure, l'analyse du destin, 30000 yens, une demande d'audience avec Asahara, 20000 yens, tout était défini dans un système de tarifs. Même si l'on regarde les choses avec bienveillance, on peut peut-être se dire qu'il y a des choses qui sont clairement bonnes, mais cela donne vraiment le sentiment que tout n'est qu'argent, argent, argent "9. Les stages d'initiation, payants la plupart du temps, doivent être réalisés dans un ordre donné pour espérer atteindre l'Illumination. La presse a révélé que l'ensemble de ces divers stages d'initiation coûtait en moyenne dix millions de yens. " A ce momentlà, confirme Takahashi Hidetoshi, tous les shukke du pays avaient été réunis à Kamikuishiki. Une nouvelle initiation allait commencer. On l'appelait Pa-fekuto.saruveshon.inishe-shon ${ }^{10}$, ou PSI en abrégé. On devait avoir le casque. Cette initiation était d'abord destinée aux shukke, mais elle était aussi offerte aux zaike qui donnaient une offrande. Celle-ci s'élevait de dix millions de yens "11. Le casque, obligatoire dans certaines circonstances, était, dit-on, vendu aux disciples dix millions de yens ou, pour ceux qui n'avaient pas les moyens de payer ce prix, loué pour un million de yens par semaine. " Le shukke que j'étais devenu devait immédiatement suivre la PSI. En fait, Aum Shinrikyô avait une variété infinie d'initiations. Une initiation, cela pouvait être simplement une cérémonie qui marquait l'entrée. On aurait pu parler de rite de passage, mais pour Aum Shinrikyô, cela s'appelait higidenju [initiation secrète]. Le gourou Asahara Shôkô était le seul à détenir des secrets qu'il transmettait rituellement à ses fidèles. Peu à peu, il y eut une sur-utilisation de certains mots. Dès que Asahara Shôkô faisait quelque chose, ça devenait tout de suite une initiation ${ }^{12}$ ".

Les rites et cérémonies, très nombreux, étaient payants. Les preuves qui sont d'ores et déjà apportées lors des procès semblent conforter la véracité de ces faits. Mais il n'est pas certain que ces tarifs aient toujours été appliqués également. Certaines pratiques étaient particulièrement coûteuses. Ainsi, l'eau du bain du gourou était distribuée à raison de trois cent mille yens le verre. Après avoir offert un million de yens, on pouvait aussi boire une dose du sang du Maître, pour acquérir des pouvoirs surhumains. Le sang du gourou était censé contenir une formule ADN très spécifique, ce qu'aurait attesté la faculté de médecine de l'Université de Kyoto. L'avocat Sakamoto, s'étant renseigné auprès de la faculté en question, s'était entendu répondre qu'il n'y avait rien de particulier à signaler concernant le sang du gourou. Un disciple, après avoir fait une offrande de un million de yens, avait bien bu le sang, mais n'en avait retiré aucun pouvoir particulier : il demandait, par l'intermédiaire de Sakamoto, que son offrande lui soit rendue.

De ces différentes manières, la secte a pu se constituer un important pactole, exonéré d'impôts. Le gourou pouvait alors offrir quelques plaisirs supplémentaires à la secte. Fin 1992, il achète un orchestre constitué de cent vingt musiciens russes dirigés par un certain Vladimir Koudria. L'orchestre baptisé Chyren ${ }^{13}$ est chargé de composer de la " musique astrale "14, destinée à être jouée exclusivement au cours d'un choix précis des rites et cérémonies de Aum Shinrikyô.

C'est au cours de cette période, finalement plutôt prospère, qu'apparaît la violence.

Les premières victimes

Des plaintes commencent à s'accumuler et quelques cris d'alarme sont lancés: ils seront très longs à porter leurs effets. 
27 Déjà, en septembre 1988, avait disparu un disciple, Mashima Teruyuki. Il semble que sa mort ait été plus accidentelle que préméditée. Il serait décédé des suites d'un entraînement trop violent pour sa constitution physique. En février 1989, par contre, sans qu'il n'y ait plus aucun doute aujourd'hui, on peut parler d'exécution pour Taguchi Shuji, supprimé pour avoir critiqué les nouvelles orientations du dogme, les méthodes d'entraînement, et avoir fait part de son intention de quitter la communauté. Peut-être cet événement peut-il encore être considéré comme un tragique fait divers.

L'affaire Sakamoto fait partie des grands crimes orchestrés par la secte. Sakamoto Tsutsumi est cet avocat de Yokohama auquel il a déjà été fait allusion précédemment. En 1989, il représente les intérêts d'un groupe de familles. Celles-ci ont un point commun : elles attaquent la secte soit parce qu'elles ne peuvent obtenir de nouvelles d'un ou de plusieurs de leurs proches devenus shukke, soit parce qu'elles souhaitent récupérer des sommes qu'elles jugent indûment données à la secte par elles-mêmes ou par un de leurs parents. Sakamoto avait mené son enquête et se trouve, fin 1989, l'un des rares à connaître les pratiques de la secte. Une journaliste, Egawa Shôko, avait mis en relation l'avocat et une des familles à l'origine de l'association des plaignants. Au cours de la même année, des reporters de la chaîne de télévision TBS enregistrent une interview du jeune avocat sur les reproches et critiques qu'il formule à l'encontre de Aum Shinrikyô. Les dirigeants de la secte, probablement informés de l'existence de cet enregistrement par un disciple travaillant au sein de TBS, se rendent dans les locaux de la chaîne de télévision, se font projeter en privé la cassette vidéo et obtiennent, à moins qu'ils ne négocient ${ }^{15}$, la déprogrammation de l'émission. Faisant leur rapport au gourou, ils reçoivent l'ordre de ramener Sakamoto dans les locaux de la secte. Celle-ci entrait alors en campagne électorale, les élections devant avoir lieu quelques mois plus tard. Les dirigeants planifient l'enlèvement de l'avocat, ratent à plusieurs reprises leur victime et, ne parvenant pas à surprendre l'avocat seul, en novembre 1989, ils le kidnappent avec sa femme et son fils alors âgé d'un an. Les victimes sont emmenées à Kamikuishiki où les deux adultes sont étranglés et l'enfant étouffé. Les corps sont ensuite enterrés dans trois sites montagneux différents. Les disparitions sont de suite signalées à la police. Assez vite, les collègues du cabinet d'avocats de Sakamoto soupçonnent Aum Shinrikyô. Des mouvements religieux fortement opposés à Aum Shinrikyô dénoncent publiquement l'organisation de Asahara Shôkô comme coupable. Pendant un mois, la police se montre sourde aux appels des avocats qui la poussent à mener une enquête auprès de la secte. Un de ses badges a bien été trouvé dans l'appartement de la famille, mais cela ne suffit pas à la police pour ouvrir une enquête. Il est bien établi que Sakamoto était en charge de plaintes déposées par un groupe de parents de disciples de la secte, qu'il s'apprêtait aussi à entamer une action juridique pour demander à la secte de rendre à un ancien disciple un don forcé de un million de yens, mais rien n'est fait. Une journaliste, Egawa Shôko, se met au travail et publie début 1991 un des tout premiers textes sur la secte. Elle y relate les circonstances, telles qu'elles sont connues alors, de la disparition de la famille Sakamoto et fait part de ses doutes, mettant en relation ces disparitions et les comportements de Aum Shinrikyô, dénonçant notamment le rapport de la secte à l'argent et son système d'offrandes. Elle soupçonne aussi la secte de développer une action d'opposition à la société ${ }^{16}$.

Les collègues de Sakamoto organisent des rassemblements dans tout le pays et demandent à la population toutes les informations qu'elle pourrait détenir sur les Sakamoto. Ils parviennent à mobiliser peu à peu l'ensemble de la profession qui 
organise différentes manifestations. La mère de l'avocat disparu parvient à entraîner toute une partie de l'opinion qui réclame l'élucidation de ce cas. L'enquête n'aboutira qu'en 1995. Tout le monde se demandera pourquoi cette enquête n'a pas été conduite plus efficacement. Incompétence ou volonté délibérée de ne pas intervenir ? Difficulté de coopération entre diverses équipes policières? Ne s'agit-il pas plutôt de la peur d'être accusé de persécuter une organisation religieuse? La police, voire l'Etat, craignaient-ils d'être identifiés à un autoritarisme déjà connu au Japon? Mais des soupçons lourds pèsent : à quels niveaux la secte bénéficiait-elle d'appuis suffisamment puissants pour freiner, voire obstruer l'enquête? N'aurait-elle pas infiltré les strates les plus élevées du pouvoir? En septembre 1995, Kunimatsu Takaji, le chef de la police nationale, reconnait publiquement que si la police avait réagi plus rapidement dans l'affaire Sakamoto, les attaques au gaz de Matsumoto et de Tokyo ne se seraient peutêtre pas produites.

Les corps sont retrouvés en septembre 1995, presque six ans après les faits, après l'attentat de Tokyo, et après que les disciples directement impliqués et emprisonnés aient informé précisément la police. Six dirigeants de la secte sont inculpés du meurtre de la famille : Okazaki Kazuaki, Murai Hideo, Ministre de la Science et de la Technologie de Aum Shinrikyô, assassiné en avril 1995, Hayakawa Kiyohide, Nakagawa Tomomasa, Niimi Tomomitsu et Hashimoto Satoru. Hayakawa et Nakagawa reconnaissent leur participation aux meurtres. Nakagawa, qui a injecté aux deux adultes du chlorure de potassium, avoue les avoir étranglés et aidé à étouffer l'enfant. Il confirme que cinq autres disciples ont participé aux meurtres. Lors de son procès, il plaide coupable pour les charges qui pèsent sur lui en relation avec cette affaire et déclare que l'enlèvement, les meurtres et l'ensevelissement des corps ont été ordonnés par Asahara Shôkô.

31 C'est enfin à cette même époque que sont déposées les premières plaintes contre la secte, émanant soit du voisinage, soit de familles de shukke. Après l'attentat de Tokyo, on recensera une centaine de plaintes contre la secte. Des parents accusent leurs enfants, tel ce couple qui dépose plainte contre sa fille qui, ayant rejoint la secte, avait retiré trente millions de yens des économies familiales.

On peut donc précisément dater le passage à la violence meurtrière de la secte : février et surtout novembre 1989, si l'on considère la mort de Mashima Teruyuki, en 1988, comme accidentelle. A ce stade, plusieurs constats doivent être faits. Le premier concerne la nature de l'affaire Sakamoto. Cette affaire aurait pu être interprétée comme un acte isolé, ne prenant sens que par rapport à une conjoncture bien précise : le souci de ne pas émettre de bruit autour de la secte au moment où elle cherche à établir de nouveaux rapports sociaux à travers son action politique. A posteriori, il nous semble que l'affaire Sakamoto est à lire dans un contexte plus global. Cette affaire, loin d'être isolée, marque au contraire l'entrée dans l'engrenage de la violence, entrée que justifie bien plus la reprise en main du dogme, des pratiques et des ambitions religieuses de la secte que l'événement particulier que représente la campagne électorale qui, elle-même, ne fait sens que dans ce cadre: la diffusion la plus large possible des croyances de la secte, la mise en place d'un monde d'un autre niveau, ni humain ou inhumain, ni terrestre ou extra-terrestre, ni actuel ou futur, un monde uniquement conçu dans l'ordre du religieux. Le second constat porte sur la responsabilité de la secte. Dans l'affaire Sakamoto, seuls le gourou et six de ses disciples sont impliqués. Et il est très probable qu'ils aient été pratiquement les seuls à être dans 
le secret, la secte n'étant pas un groupe unifié dans lequel tous les disciples, et notamment tous les shukke, auraient été prêts à justifier de telles actions. façon pensée, ordonnée et contrôlée. Pourquoi cette année-là plutôt que dès 1984-1985, ou plutôt qu'à un autre moment? Asahara shôkô et quelques-uns de ses proches se rendent en Russie, la secte s'implante à l'étranger. Est-ce la rencontre avec la Russie qui permet de passer à des moyens armés ou bien est-ce parce que la secte avait le projet de s'armer qu'elle établit des liens avec la Russie? Sans pouvoir apporter de réponse, on ne peut que se poser ces questions. La secte a de l'argent et commence à être bien établie. Elle a sans doute ses problèmes internes : des membres importants veulent partir, des tensions entre certains leaders brouillent la hiérarchie du pouvoir, une relative stagnation marque les recrutements. Peut-être aussi la secte s'installe-t-elle dans la paranoïa, persuadée que d'autres mouvements cherchent à l'affaiblir, quand ce n'est pas la société japonaise ou les Américains. S'il n'est pas impossible que ces différents facteurs aient pu pousser les dirigeants vers une action violente, une autre explication doit être envisagée, à partir, plus fondamentalement, des croyances de la secte, c'est-à-dire de ses raisons d'être. Armagedon approche: il est temps de se préparer pour la Troisième Guerre mondiale au cours de laquelle Aum Shinrikyô pense avoir une mission à remplir vis-à-vis de l'humanité. C'est au nom de ces croyances que les shukke ont tout abandonné; ce sont ces croyances qui rassemblent les dirigeants autour de leur gourou et qui les mobilisent, inébranlables, sans doute solidement intériorisées à travers le travail de la communauté et l'intensité des pratiques et des rites. Ils continuent à avoir des activités à l'extérieur de l'organisation : ils se déplacent, dirigent des entreprises, rencontrent des gens, achètent, vendent et négocient. Mais toutes ces activités sont entièrement mises au service de leurs croyances et de leur Maître. En 1989, Asahara Shôkô publiait deux textes sur l'Apocalypse de Saint-Jean ${ }^{17}$. L'idée de l'apocalypse n'est pas neuve pour le gourou qui s'y référait à travers la légende du royaume de Sambhala dirigé par la déesse Siva protectrice de la secte. Selon la légende, tout se termine dans une guerre finale. Seul subsiste un "Royaume millénaire ", et Asahara Shôkô ajoute que la mission qu'il a reçue lui donne l'ordre d'établir ce royaume au Japon avec lui-même à sa tête. Aum Shinrikyô pense en effet pouvoir trouver les moyens de faire en sorte que le Japon soit le royaume de Sambhala. Ainsi, les pouvoirs sacrés de la secte vont s'étendre à l'ensemble du pays, et c'est pourquoi l'humanité pourra être sauvée à partir du Japon. Le seul moyen d'atteindre le bonheur dans un monde futur est donc de rejoindre Aum Shinrikyô et de l'aider dans sa mission. 
38 La date à laquelle la prophétie doit se réaliser est peu à peu avancée et précisée : annoncée dans un premier temps aux alentours de l'an 2000, elle doit toucher notre monde plus tôt: début 1997, puis fin 1996, puis en novembre $1996^{18}$. La chute du communisme soviétique est vue comme un signe de l'approche de l'apocalypse :

" L'apogée du matérialisme comme résultat de l'expansion des désirs de l'homme sur terre va rencontrer l'apogée du spiritualisme. Regardons la situation d'aujourd'hui : nous avons atteint l'apogée du matérialisme : le socialisme s'est effondré et demeure seulement le matérialisme. J'ai fait la prédiction suivante, celle de la rencontre entre les matérialistes authentiques et les spiritualistes authentiques. Maintenant qu'il n'y a plus de socialisme, ça va se réaliser. Les spiritualistes authentiques vont pleinement se développer et éclater comme le soleil, tandis que les matérialistes authentiques seront rassemblés pour être brûlés "19.

$40 \mathrm{Au}$ cours de conférences qu'il donne en octobre et novembre 1992 dans plusieurs universités de renom, Asahara Shôkô prédit qu'Armagedon arrivera avant l'an 2000, que les " armes $\mathrm{ABC}$ " [atomiques, biologiques et chimiques] détruiront $90 \%$ de la population urbaine et que le seul moyen de survivre sera de devenir " superhumain ", ce que seul permet un entraînement spirituel ${ }^{20}$. Il faut donc échafauder différents plans pour assurer la survie de ceux qui seront sauvés: on pense au sein de la secte à des villes sous-marines, par exemple. Il va falloir s'entraîner à supporter la chaleur terrible qui sera produite à Armagedon, d'où les pratiques d'immersion dans l'eau chaude qui font l'objet d'un entraînement intensif et dont le but est d'arriver à rester dans une eau à $50^{\circ} \mathrm{C}$ pendant une quinzaine de minutes ${ }^{21}$. Il faut se mettre dans des conditions propres à se défendre, c'est-à-dire trouver les moyens de se procurer des armes équivalentes à celles qui seront utilisées. Le thème de la Troisième Guerre mondiale prend de la sorte de plus en plus de place et finit par absorber tous les autres. Aum Shinrikyô dramatise son enseignement et l'imminence de la fin dernière définit, à ses yeux, l'ordre des priorités. Comme la secte pense être seule à avoir conscience de sa mission, de son rôle envers la survie de l'humanité, de l'urgence de la situation et de l'ampleur de sa responsabilité, elle ne s'estime redevable qu'envers les dieux qui lui ont transféré des pouvoirs surnaturels. La venue de la fin dernière, considérée comme proche et certaine, justifie pour les dirigeants de la secte et un entraînement spirituel et physique intense et l'armement de leur groupe. Il n'est plus aucune tangibilité qui puisse dès lors freiner l'emballement d'une machine sectaire que l'intensité des pratiques et le discours religieux ont entraînée dans un monde imaginaire et tenue recluse. Le thème de la " dernière guerre " met au premier plan des idées qui deviennent omniprésentes: l'immortalité et la mort, mort que l'on désigne par le terme Pho-wa, " pratique [qui] vous donne la possibilité de vous réincarner dans le royaume de votre choix au moment de votre mort "22. Et, à partir de 1994, s'entraînant avec une rigueur vitale pour la survie spirituelle, psychologique et physique, les shukke se livrent à un ascétisme extrême, ascétisme explicitement rendu nécessaire par l'approche de la Troisième Guerre.

Ne compter que sur ses propres moyens

L'armement de la secte est conçu et programmé à partir de 1992 et les attentats au gaz de Matsumoto et de Tokyo sont la suite du plan élaboré par la secte, même si le choix des moyens de ces attaques, les moments, les lieux et les cibles sont arrêtés juste avant les faits, même si ce sont d'autres événements qui déclenchent au dernier moment le passage à l'action. 

explosif. un seul et même plan.
Par tous les moyens, la secte cherche à s'armer, alliant la recherche, l'appui sur les réseaux relationnels existants et la constitution de nouveaux, la production et l'achat sur des marchés parallèles. Il s'agit de répondre aux armes " $\mathrm{ABC}$ " par des armes de capacité équivalente et, outre les armes classiques, la stratégie de la secte concerne par conséquent les armes biologiques et chimiques, et plus difficile et incertain encore, les armes atomiques. Un tel objectif suppose qu'en amont, la secte ait pu réunir les fonds nécessaires autant que les compétences scientifiques et militaires. Il faut probablement qu'elle bénéficie de soutiens importants dans le monde politique et administratif et qu'elle ait des membres capables d'établir des relations avec les réseaux adéquats.

Un tel programme ne peut être le fait d'une simple lubie d'un gourou qui aurait perdu tout contact avec le principe de réalité. Certes, Asahara Shôkô prend, ou entérine, toutes les décisions importantes. Ainsi, il décide, avec quelques-uns de ses lieutenants, fin 1992, de commencer par la production d'une série de mille fusils automatiques ${ }^{23}$ et de un million de munitions. La secte s'est procuré quatre-vingt machines-outils capables de produire les pièces : un des disciples a fait don de son entreprise et de ses machines. Le programme doit démarrer au printemps 1993 et s'achever deux ans et demi plus tard, en automne 1995. Au sein de Satyam 11, une usine est rapidement construite. Il en sort un premier prototype de fusil automatique entre novembre 1994 et janvier 1995, un second deux mois plus tard. Le programme restera inachevé : la police saisira, après l'attentat de Tokyo, les machines et plusieurs milliers de pièces et des munitions. La secte n'avait pas eu le temps de procéder à l'assemblage.

Deux disciples suivent un stage de pilotage d'hélicoptère en Floride et obtiennent leur licence en 1993. Aum Shinrikyô recherche des petits hélicoptères, comme ceux que l'on utilise pour répandre des engrais. On retrouvera dans les notes des suspects des indications de prix, dont ceux d'une entreprise française: " 100000 \$ ", entreprise probablement contactée par la secte, mais qui n'a pas donné suite. Ils finissent par se procurer deux petits appareils autoguidés ${ }^{24}$, avec l'intention, selon la presse, de diffuser du gaz. Mais les deux appareils sont rapidement accidentés.

Des renseignements sont également collectés sur le prix de tanks, ce qu'attestent les notes du responsable de l'Agence de Défense de la secte, Kibe Tetsuya: tanks russes d'occasion, entre deux cent et trois cent mille yens, un million de yens si les engins sont munis d'un moteur neuf. La secte manie aussi les explosifs. Le 16 mai 1995, quelques heures après l'arrestation de Asahara Shôkô, le gouverneur de Tokyo reçoit un colis piégé : son secrétaire est gravement blessé à sa place. Par ailleurs, la fabrication de TNT intéresse la secte, qui a pu en produire une petite quantité, et l'on retrouvera des plans pour le financement d'un programme de production de trente mille tonnes de cet

C'est exactement à la même époque, fin 1992, que se concrétise l'idée des armes chimiques. Produire des gaz toxiques et fabriquer des fusils automatiques constituent

Les informations scientifiques nécessaires à la fabrication du sarin sont rapportées des Etats-Unis par une disciple en 1992. Les recherches sur ce gaz sont rapidement mises en oeuvre, en mars 1993. Au cours de cette période d'essais et d'expériences, plusieurs disciples sont intoxiqués et traités par Hayashi et les médecins de la secte qui se sont procurés, par des intermédiaires militaires, les informations sur l'antidote du sarin. 
La cinquième et dernière phase de la production semble en effet la plus dangereuse. Mais, au bout de quelques mois, vers la fin octobre, les recherches se soldent par de premiers " succès ": Tsuchiya a fabriqué vingt grammes de sarin. Quelques jours plus tard, avec trois autres disciples, chimistes eux aussi, il en produit six cents grammes. Le processus de fabrication est désormais considéré comme maîtrisé. Tsuchiya Masami a alors vingt-huit ans. Il a étudié la chimie organique à l'Université Tsukuba. Il agit sous les ordres de Murai Hideo, Ministre de la Science et de la Technologie. Ce dernier est directement placé sous l'autorité du gourou. Le croisement des sources et des témoignages au moment des procès établissent que Asahara Shôkô a donné l'ordre en mars 1993 à Murai de faire ce qu'il fallait pour produire massivement des gaz toxiques. Tsuchiya est à la hauteur : il devient responsable de la Division Chimie du Ministère et développe ses recherches parallèlement sur plusieurs types de gaz toxiques. Il rend ses conclusions : le sarin est le gaz le plus facile à fabriquer, notamment parce que les matières premières peuvent être acquises sans grande difficulté. Trois responsables, Endo Seiichi, Ministre de la Santé, Hayashi Ikuo, " Ministre des Traitements ", et Tsuchiya Masami proposent alors un plan au gourou : le sarin pouvait être un moyen de lutte pour Armagedon. Asahara Shôkô en ordonne alors la fabrication d'une très grande quantité. En février 1994, les premiers trente kilos sont stockés et, au même moment, la secte achète, pour 345 millions de yens, diverses machines destinées à une fabrication à plus grande échelle. Au début du mois de juin de la même année, vingt kilos supplémentaires sortent de l'usine chimique.

L'attentat de Matsumoto a lieu le 27 juin. C'est un test grandeur nature. Il permet de vérifier non seulement la qualité du gaz et ses effets, mais aussi la procédure de diffusion. Un autre disciple, Takizawa Kazuyoshi, alors âgé de vingt-quatre ans, reçoit la mission du porte-parole de Aum Shinrikyô, Joyu, de construire l'usine qui servira à effectuer le programme de fabrication du sarin. Sur le site de Satyam 7, dont le chantier est ouvert depuis l'été 1993, à Kamikuishiki, s'élève un bâtiment de trois étages, d'une surface de $1.500 \mathrm{~m}^{2}$. Le programme prévoit soixante-dix tonnes à produire au rythme de deux tonnes par jour. L'usine est en état de fonctionnement à partir de septembre 1994, date à laquelle commence la production du sarin. Bien qu'elle tourne 24 heures sur 24 avec deux équipes, le programme prend vite un très grand retard. Plusieurs disciples sont intoxiqués par les émanations. Tsuchiya joue un rôle capital dans le processus de production. Il y croit : il a d'ailleurs publiquement " prédit " que des gaz comme le sarin seraient utilisés lors de la Troisième Guerre mondiale. Il travaille sur ce projet en liaison très étroite avec Endo Seiichi, trente-deux ans, Ministre de la Science et de la Technologie, et avec Watanabe Kazumi, trente-quatre ans, Vice-Ministre de la Science et de la technologie. Ce dernier est diplômé de l'Institut de technologie de Tokyo et a travaillé comme designer pour ordinateurs dans une entreprise électronique. Ces dirigeants ont des responsabilités au sein de la secte qui les place automatiquement dans le cercle étroit qui approche facilement le gourou. Leurs connaissances scientifiques ne peuvent leur laisser aucun doute sur la toxicité de ce qu'ils sont en train de produire. Enfin, leurs fonctions au sein de la secte supposent la solidité de leur croyance dans ce qui constitue le coeur du dogme, à savoir la mission de sauver l'humanité d'Armagedon. Il est probable que les autres disciples travaillant dans la division chimique n'aient pas eu d'information sur les projets d'utilisation du sarin. De son côté, la police fait des prélèvements dans le sol autour des bâtiments de Kamikuishiki. Les analyses révèlent en novembre 1994 la présence de diverses substances chimiques qui sont celles que laisse le sarin quand il se décompose. 
Quelques mois plus tard, en janvier 1995, la secte décide de démanteler l'usine et de la transformer en lieu de culte. Deux hypothèses seront plus tard avancées par les observateurs. La première est que Aum Shinrikyô cesse la production du sarin à cause de difficultés techniques (incidents trop nombreux dans le processus de fabrication, intoxications sévères, risques considérables et incapacité à suivre le programme de production). Cette hypothèse laisse cependant dans l'ombre la raison du démantèlement rapide de l'usine. Il est possible aussi, deuxième hypothèse, que la secte ait été informée d'une probable intention de la police de perquisitionner sur le site de Kamikuishiki dans le cadre des différentes plaintes qui avaient été déposées.

$\mathrm{Au}$ sein de la même structure, parfois avec les mêmes spécialistes, des recherches concernant la production d'autres types de gaz toxiques sont poursuivies. Ainsi en estil du gaz VX, qui avait été développé en Angleterre en 1950. Comme le sarin, le VX, dont la toxicité est considérée comme cent fois supérieure, provoque des difficultés de respiration et la mort. Le processus de production de ce gaz est proche de celui du sarin. C'est aussi en novembre 1993 que Tsuchiya parvient à produire les premières doses de VX. Au début de l'été 1994, Asahara Shôkô ordonne la fabrication d'un kilogramme de VX. La secte utilisera le VX pour agresser plusieurs personnes isolées, mais elle en dispersera aussi dans la banlieue de Matsumoto en juin 1994 et dans le métro de Tokyo en mars 1995.

53 Après l'attentat du métro de Tokyo, des disciples poursuivent leur action, avec trois nouveaux attentats qui échouent à Tokyo, l'un le 5 mai 1995 à Shinjuku, les deux autres, le 4 juillet 1995, à Kabayachô, puis de nouveau à Shinjuku. Cet endroit, à la fois quartier d'affaires, centre commercial et lieu de plaisir, est le plus fréquenté de la capitale, le plus animé autant en surface que sous terre où une véritable ville souterraine a été construite sous la gare et les environs. Une quantité de cyanhydrure suffisante pour éliminer dix mille personnes dans chaque cas est trouvée. Les appareillages de diffusion du gaz n'ayant pas fonctionné, il n'y a pas de victime. Et, en octobre 1995, 8,5 kg de cyanure de sodium sont saisis par la police qui estime qu'une telle quantité aurait pu tuer soixante-dix mille personnes.

54 La secte aurait également produit plusieurs centaines de kilogrammes d'ypérite, gaz qui est plus connu sous le nom de gaz moutarde, en raison de son odeur, qui provoque des lésions cutanées et attaque les yeux et les poumons. D'autres gaz encore, qui n'ont pu être identifiés de manière certaine, semblent avoir été utilisés par la secte. Ainsi, dans le métro de Yokohama, cinq cents personnes sont intoxiquées le 19 avril 1995, et plusieurs dizaines le 2 juillet 1995 et, le 3 juillet, dans un grand magasin de la même ville, six autres personnes incommodées par des inhalations doivent être hospitalisées. Mais dans ces dernières affaires, la culpabilité de la secte n'est pas établie de manière sûre.

55 En plus des gaz toxiques qu'elle expérimente et produit, Aum Shinrikyô fabrique, à Kamikuishiki, pour son usage propre, diverses substances, sous la responsabilité du Ministre de la Santé, Endo, qui conserve ces produits et contrôle leur diffusion. Notamment, 1,7 kg de penthotal ou " sérum de vérité " a été fabriqué entre novembre 1994 et février 1995. Ce produit aurait été utilisé pour pratiquer des lavages de cerveau sur les disciples récalcitrants et sur ceux qui tentaient de partir. Mais aussi, la secte pense à ce moment que la police a infiltré leur mouvement: la traque aux espions occupe l'esprit de certains dirigeants. On sait par ailleurs que c'est à la suite d'une injection de penthotal que le notaire Kariya a succombé, sans qu'il y ait eu 
nécessairement intention de tuer. Vers la mi-1993, la secte commence à avoir recours de plus en plus souvent à divers stimulants, parfois sous formes d'injections. Sont produits et utilisés différents hallucinogènes, comme la mescaline dont à peu près vingt mille doses sont retrouvées, des amphétamines ou de grandes quantités de LSD. Ces drogues sont surtout utilisées au cours de rites religieux ${ }^{25}$.

$\mathrm{Au}$ moment de la guerre finale, les armes chimiques ne seront pas les seules à être utilisées. Il y aura aussi, dit le gourou à ses disciples, des armes bactériologiques. La secte explore dans cette direction. Elle se procure donc des matériels spécialisés et réalise des expériences sur des combinaisons de gènes et de cellules avec le projet de tenter de produire des virus. Ainsi, des bacilles du botulisme sont répandus, sans conséquence sérieuse, pendant l'été 1993, autour du Palais Impérial. Un des disciples témoigne que des appareillages destinés à diffuser ces bactéries ont été installés à Kasumigaseki, quelques jours avant l'attentat. En juin 1993, les résidents de l'arrondissement de Koto, à Tokyo, constatent une odeur étrange, éventuellement liée à la production de bactéries en rapport avec l'anthrax. A la même époque, la secte tentait de faire croire qu'elle était victime d'attaques bactériologiques de la part des Américains. La secte justifie l'étrange odeur : ce ne sont que des haricots de soja que l'on fait brûler avec diverses essences pour les rites religieux. Peut-être plus inquiétant encore le fait qu'une équipe médicale d'Aum Shinrikyô se soit rendue au Zaïre au moment où le virus Ebola y décimait une partie de la population. Sur le temps d'antenne que la secte avait acheté sur une radio de Moscou, Endo affirme alors à ses auditeurs que le virus Ebola peut être utilisé comme une arme, tout comme la variole, la fièvre jaune ou le botulisme. Lors d'une discussion radiodiffusée, Asahara Shôkô et Endo Seiichi expliquent comment de telles armes pourraient être utilisées au moment de l'apocalypse, telle que prévue par le gourou. Ces armes sont présentées comme des menaces pour la secte et non comme pouvant être utilisées par elle.

Hayakawa Kiyohide, un des personnages-clés dans plusieurs des affaires qui impliquent la secte, est considéré comme le numéro deux de l'organisation religieuse. Agé de quarante-cinq ans en 1995, il est le dirigeant le plus âgé. La police saisit plusieurs feuilles de notes lui appartenant dans lesquelles sont inscrits des tarifs, des contacts, des renseignements précis concernant des armes à laser, mais aussi du minerai d'uranium. Il s'était aussi rendu en Australie en avril 1993, où il avait rencontré des responsables d'entreprises dont la spécialité était en relation avec ce minerai.

C'est donc en 1992 que ces programmes de recherche et de production sont conçus. C'est aussi au cours de cette année 1992 que la secte essaime à l'étranger, et de manière plus spectaculaire en Russie, pays où le fait religieux connaît alors un regain spectaculaire. La concordance des dates ne suffit certainement pas à valider une hypothèse. Ainsi, rien ne permet d'affirmer à partir des informations qui ont pu être collectées que la rencontre de la secte avec la Russie déclenche le passage du mouvement vers la violence ${ }^{26}$.

59 Aum Shinrikyô a alors gagné en assurance sur le plan spirituel, notamment avec le séminaire d'Ishigakijima et ses conséquences, et son assise financière paraît solide. Commence l'extension du mouvement à l'extérieur du Japon. En 1992, la secte s'établit presque simultanément en Russie, en Europe, à Bonn, aux Etats-Unis et à Colombo, au Sri-Lanka. La branche russe est celle qui progresse le plus, au point de compter rapidement un nombre d'adeptes russes plus important que le nombre de disciples japonais. En trois ans, Aum Shinrikyô a recruté trente mille adeptes russes, ouvert six 
différents sites à Moscou et un autre plus au sud, à Vladikavkaz. Elle a établi onze branches en dehors de la capitale, à Saint-Pétersbourg, Vladivostok, Vorkuta dans l'Oural et dans plusieurs autres villes. La secte prêche en Russie, notamment à travers Radio Mayak, avec une heure quotidienne d'antenne. Quelques semaines après l'attentat du métro de Tokyo, les autorités russes demandent la fermeture des représentations de la secte sur leur territoire.

D'après les enquêteurs, les dirigeants de Aum Shinrikyô ont rencontré des autorités scientifiques russes en 1992 et 1993. A Moscou, en 1992, Asahara Shôkô lui-même se serait entretenu avec Nikolai Basov, prix Nobel de physique, qui a notamment mis au jour les principes du laser. La branche moscovite de la secte aurait proposé une rencontre entre le gourou et le Ministre de l'Energie Nucléaire, Viktor Mikhailov. La presse révèle aussi que la secte se serait procurée sur place une vingtaine d'exemplaires d'un manuel d'entraînement militaire. Un autre lieu de passage a sans doute été important pour la secte : l'Institut de physique nucléaire Kurchatov. Andrei Gagarinsky, porte-parole de l'Institut, confirmera qu'au moins un des chercheurs est disciple de Aum Shinrikyô. Pendant l'année 1993, plusieurs dirigeants de la secte effectuent des séjours très fréquents en Russie ou dans des Républiques de l'ex-bloc soviétique. Un des faits les plus étonnants est l'achat d'un très gros hélicoptère, de type MIL Mi-17. Un tel appareil pèse sept tonnes, peut embarquer vingt-huit personnes et voler à $240 \mathrm{~km} / \mathrm{h}$. Il peut être équipé d'armes puissantes comme des missiles ou des roquettes. Des dirigeants de la secte se le seraient procuré en Azerbaïdjan, durant le printemps 1994. Une entreprise autrichienne se serait ensuite chargée d'effectuer les transactions, de démanteler l'appareil et de l'envoyer par bateau à partir des Pays-Bas, après avoir fait transiter les pièces par la Slovaquie. Arrivées au port de Yokohama en juin 1994, les pièces passent apparemment sans difficulté la douane où les dirigeants d'Aum Shinrikyô procèdent aux formalités, avant d'être transportées à Kamikuishiki où les disciples qualifiés procèdent au remontage de l'appareil. Une quinzaine de shukke, dont Hayakawa, suivent un entraînement au tir au sein d'une unité militaire de la région de Moscou. Probablement sur une des bases d'infanterie proches de la capitale, Kantemirovskaya ou Tamanskaya. Ils y restent une dizaine de jours en septembre 1994. Bien après l'attentat de Tokyo, les autorités russes reconnaissent que le Général Leonty Kynetsov, responsable de la région de Moscou, a effectivement donné son autorisation.

\section{NOTES}

1. Cf. Takahashi Hidetoshi, Aomu kara kikan [Retour d'Aum Shinrikyô], Tokyo, 1996, p. 38.

2. Asahara Shôkô, Beyond life and Death, 1993, p. 56.

3. Cf. Shimazono Susumu, " In the Wake of Aum Shinrikyô ", Journal of Religious Studies, 1995, 22 /3-4, 381 : 415, p. 395.

4. Asahara, Declaring myself the Christ, op. cit., p. 123. 
5. Satyam provient du sanskrit et signifie vérité.

6. En effet, très souvent, l'entrée dans la secte comme shukke est concomitante avec la déstructuration de la famille, qu'elle en soit la cause ou l'effet. Plusieurs mères sont entrées dans la secte avec leurs enfants et un divorce s'en est suivi. Les pères ayant perdu la trace de leurs enfants ont fini par abandonner toute recherche et tout désir de les revoir.

7. Plusieurs dirigeants de cette entreprise seront arrêtés en juin 1995... pour piratage de logiciels.

8. Cf. Takahashi Hidetoshi, op. cit., p. 91.

9. Cf. Egawa Shôko, Kyseisha no yabô [Ambitions pour un Messie], Tokyo, Kyôiku shiryô shuppankai, 1991, p. 84.

10. C'est-à-dire la transcription japonaise de l'anglais Perfect Salvation Initiation.

11. Cf. Takahashi Hidetoshi, op. cit., p. 75.

12. Cf. Takahashi Hidetoshi, op. cit., p. 119.

13. Soit " offrande divine " en sanskrit.

14. Sans entrer ici dans le détail des croyances religieuses de Aum Shinrikyô, précisons que les fidèles devaient successivement passer dans ce qu'ils appellent le monde astral, puis dans le monde causal, chacun de ces mondes étant divisé en plusieurs niveaux à gravir l'un après l'autre, avant d'espérer pouvoir parvenir au gedatsu.

15. Il est probable que la non-diffusion de l'interview ait été négociée contre une interview exclusive que Asahara Shôkô aurait accordée à la chaîne TBS à Bonn où il se trouvait avec plusieurs de ses disciples fin 1989.

16. Cf Egawa Shôko, Kyûseishu no yabô, op. cit.

17. Asahara Shôkô, Metsubô no hi [Le jour de la fin du monde], Tokyo, Aum Shinrikyô Shuppan, 1989 ; Metsubô kara kokû he : zoku metsubô no hi [De la fin dernière au monde vide : les suites de la fin du monde], Tokyo, Aum Shinrikyô Shuppan, 1989.

18. D'autres sectes importantes au Japon annoncent la fin prochaine du monde : Tôitsu Kyôkai, Kôfuku no Kagaku.

19. Cf. Asahara Shôkô, Declaring myself the Christ, op. cit., p. 129.

20. Shimazono Susumu, "In the Wake of Aum Shinrikyô : the Formation and Transformation of a Universe of Belief", dans Japanese Journal of Religious Studies, 1995, 22/3-4, $381: 415$, p. 402.

21. Cette pratique est justifiée de diverses manières. L'apocalypse va être accompagnée d'un déluge d'eau chaude et il faut entraîner le corps à survivre dans ces conditions. Mais les bains d'eau chaude semblent aussi considérés par la secte comme une thermothérapie et, dans certains cas, ils constituent un châtiment.

22. Cf. Asahara Shôkô, Beyond Life and Death, op., cit., p. 67.

23. Le modèle est une arme russe, type AK 74, dont au moins un exemplaire a été rapporté d'un des tout premiers voyages effectués par les dirigeants de la secte en Russie à cette époque.

24. Ces appareils ont une autonomie de vol d'environ quatre-vingt-dix minutes et peuvent voler à 85 miles $/ \mathrm{h}$; ils peuvent porter des charges d'une dizaine de kilogrammes. Ils sont généralement utilisés pour prendre des photos aériennes de sites difficilement accessibles par d'autres moyens ou pour diffuser des engrais.

25. Par exemple, au cours de " l'Initiation du Christ ", Asahara Shôkô offrait au disciple initié un verre rempli de liquide contenant du LSD. La boisson devait être absorbée tout en récitant des chants religieux. La dose avalée produisait son effet pendant une 
dizaine d'heures. Après quoi, le disciple nouvellement initié recevait des diurétiques par injection intraveineuse et devait prendre des bains d'eau chaude.

26. Les premières contacts importants ont probablement lieu au début de 1992 au Japon. A cette époque, Asahara Shôkô aurait rencontré Oleg Lobov, alors secrétaire du Conseil de sécurité. L'objet de la rencontre aurait été un projet d'université russojaponaise pour lequel Lobov recherchait des fonds lors de son voyage au Japon. En 1992, la secte avait de l'argent. Elle aurait alors fait un don d'un million de dollars pour une fondation chargée de mettre en oeuvre le projet, fondation dont Lobov était le viceprésident. Asahara Shôkô se serait ensuite rendu en Russie au printemps 1992, avec trois cents de ses disciples, invités par cette université qui n'avait d'autre existence que la fondation censée la créer. Le quotidien russe Izvestia rapportera, après l'attentat de Tokyo, que Oleg Lobov aurait facilité les activités de la secte en Russie. Toujours est-il que la secte a pu se développer très rapidement en Russie à partir de 1992.

INDEX

Index géographique : Japon

Mots-clés : religion, violence, guérillas et organisations clandestines, attentat 